\title{
Equation of state of lead from high-pressure neutron diffraction up to 8.9 GPa and its implication for the $\mathrm{NaCl}$ pressure scale
}

\author{
Th. Strässle, ${ }^{1, *}$ S. Klotz, ${ }^{2}$ K. Kunc, ${ }^{2}$ V. Pomjakushin, ${ }^{3}$ and J. S. White ${ }^{3,4}$ \\ ${ }^{1}$ Paul Scherrer Institut, $\mathrm{CH}-5232$ Villigen PSI, Switzerland \\ ${ }^{2}$ Institut de Minéralogie, de Physique des Matériaux et de Cosmochimie, CNRS - Unité Mixte de Recherche 7590, \\ Université Pierre et Marie Curie, 4 Place Jussieu, F-75252 Paris, France \\ ${ }^{3}$ Laboratory for Neutron Scattering and Imaging, Paul Scherrer Institut, CH-5232 Villigen PSI, Switzerland \\ ${ }^{4}$ Laboratory for Quantum Magnetism, Ecole Polytechnique Fédérale de Lausanne, CH-1015 Lausanne, Switzerland
}

(Received 4 March 2014; revised manuscript received 19 May 2014; published 2 July 2014)

\begin{abstract}
From high-resolution neutron-diffraction experiments we present equation-of-state (EOS) data of elemental lead as functions of both temperature (80-298 K) and applied pressure (up to $8.9 \mathrm{GPa}$ ), with the pressure values derived from the $\mathrm{NaCl}$ pressure gauge. Based on Brown's $1999 \mathrm{NaCl}$ EOS we find the bulk modulus of lead of $B_{0}=41.2(2) \mathrm{GPa}$ at $298 \mathrm{~K}$ to increase by $14 \%$ to $B_{0}=47.0(5) \mathrm{GPa}$ by $80 \mathrm{~K}$. The ambient temperature value coincides within $0.35 \mathrm{GPa}(1.7 \%)$ of the value determined from published ultrasonic data. The good agreement between the neutron and ultrasonic data deteriorates if pressure values are derived from Decker's 1971 scale. Our results hence lend significant support to Brown's revised $\mathrm{NaCl}$ pressure standard. Furthermore, the experimental results are compared with $T=0 \mathrm{~K}$ first-principles calculations.
\end{abstract}

DOI: 10.1103/PhysRevB.90.014101

PACS number(s): $64.70 . \mathrm{K}-$, 61.50.Ks, 71.30.+h, 75.50.Gg

\section{INTRODUCTION}

Lead is considered a prototype for studying the behavior of heavy elements under high and ultrahigh pressures. At ambient conditions, it crystallizes in the cubic fcc structure which transforms under pressure to a simple hexagonal close-packed (hcp) structure at $13 \mathrm{GPa}$, which then converts into a bodycentered cubic (bcc) structure at $\approx 100 \mathrm{GPa}[1,2]$. Lead is a very compressible metal and the equation of state (EOS, i.e., the pressure-volume relation) has attracted quite a lot of interest [1-5]. While the results of both $\mathrm{x}$-ray synchrotron [1-3] and ultrasonic measurements [4,5] agree on a bulk modulus of $\approx 41 \pm 2 \mathrm{GPa}$ at $300 \mathrm{~K}$, its temperature dependence is currently far less constrained. From general considerations one would expect that $B_{0}$ increases with decreasing temperature [6,7]. Indeed, early ultrasonic measurements [4] report an increase of $6 \%$ between 300 and $100 \mathrm{~K}$, but later data [5] instead evaluate the increase over this temperature range to $12 \%$. This is in sharp contrast to higher-temperature synchrotron x-ray-diffraction data from which an increase of $B_{0}$ by only $1.6 \%$ is expected [8]. From the same study, a decrease of $B_{0}^{\prime}$ with increasing temperature was also reported, which is unexpected on physical grounds. The issue has recently gained importance since lead is becoming increasingly popular for use as a pressure marker for neutron experiments in the 0-10 $\mathrm{GPa}$ range including studies at cryogenic temperatures. It is a strong neutron scatterer with negligible absorption and incoherent cross section, and its high compressibility makes pressure readings rather sensitive. Furthermore, due to its low shear strength, lead is an efficient solid pressure-transmitting medium at low temperatures. The knowledge of an accurate EOS in the 0-300-K range is therefore desirable for highpressure metrological reasons.

In this paper we report high-resolution neutron-diffraction data on the EOS of lead, at 80 and $300 \mathrm{~K}$. The pressures were

*Corresponding author: thierry.straessle@psi.ch determined using the $\mathrm{NaCl}$ pressure standard, i.e., Decker's [9] EOS with its low-temperature extension by Skelton et al. [10], as well as the more recent EOS reported by Brown [11]. All of them are believed to be accurate to $\approx 5 \%$. In this context it should be recalled that the vast majority of published equations of state have been established using secondary pressure standards. Examples include the ruby scale, which has been calibrated against the $\mathrm{NaCl}$ scale at low pressures, and shock-wave data obtained from metals at high pressures [12]. The same applies to high-pressure ultrasonic data which rely on the accuracy of manometers that are calibrated against reference devices. The calibration of the EOS of lead against $\mathrm{NaCl}$, as done in this work, is a highly useful strategy since it can be easily revised if a more accurate $\mathrm{EOS}$ of $\mathrm{NaCl}$ becomes available in the future.

\section{EXPERIMENTAL}

Several samples were prepared from carefully mixed fine powders of $\mathrm{NaCl}\left(99.5 \%\right.$, dried at $150^{\circ} \mathrm{C}$ for $\left.1 \mathrm{~h}\right)$ and lead (freshly ground from a bulk sample) in a $(\mathrm{NaCl} / \mathrm{Pb})$ mass ratio of 0.70 , pressed into pellets of $45-\mathrm{mm}^{3}$ volume, and inserted into a VX5 Paris-Edinburgh press using TiZr encapsulating gaskets. The high-pressure setup was otherwise identical to a previous experiment conducted on the same instrument [13]. No pressure-transmitting medium (PTM) was used in the experiments since lead is already an exceptionally good solid PTM [14], and the spheroidal pressure chamber of the anvils ensures a quasi-isotropic compression with very small uniaxial components [15]. The addition of a standard fluid PTM such as the 4:1 methanol-ethanol mixture would have deteriorated the hydrostatic conditions at low temperatures where these PTMs are frozen. To avoid buildup of strain in the samples, all pressure changes were carried out above $180 \mathrm{~K}$. Neutron-diffraction data were collected at the high intensity diffractometer HRPT at the Paul Scherrer Institut, Villigen (Switzerland), using a wavelength of $\lambda=1.494 \AA$. Diffraction 
TABLE I. Experimental unit-cell volumes $\left(\AA^{3}\right)$ for $\mathrm{Pb}$ and $\mathrm{NaCl}$ at 298 and $80 \mathrm{~K}$ and corresponding pressures (GPa) using $\mathrm{NaCl}$ pressure scales by Skelton et al. [10] and Brown [11,22], respectively. Corresponding errors of pressures are $\leqslant 0.02 \mathrm{GPa}$.

\begin{tabular}{rrrrr}
\hline \hline & $V_{\mathrm{Pb}}$ & $V_{\mathrm{NaCl}}$ & $P_{\text {Skel }}$ & $P_{\text {Brown }}$ \\
\hline$T=298 \mathrm{~K}$ & $121.24(1)$ & $179.34(1)$ & 0.03 & 0.03 \\
& $119.60(9)$ & $174.85(9)$ & 0.67 & 0.68 \\
& $117.47(8)$ & $169.99(6)$ & 1.48 & 1.52 \\
$117.13(4)$ & $169.48(3)$ & 1.58 & 1.61 \\
& $116.13(3)$ & $166.98(2)$ & 2.05 & 2.11 \\
& $113.50(3)$ & $161.27(3)$ & 3.31 & 3.38 \\
& $111.66(4)$ & $157.50(3)$ & 4.28 & 4.37 \\
& $110.69(4)$ & $155.22(3)$ & 4.93 & 5.04 \\
& $109.90(4)$ & $153.94(2)$ & 5.32 & 5.42 \\
& $108.12(5)$ & $150.51(3)$ & 6.44 & 6.58 \\
& $105.35(8)$ & $145.02(6)$ & 8.53 & 8.74 \\
& $119.11(1)$ & $175.40(1)$ & 0.06 & 0.05 \\
& $115.38(2)$ & $166.38(1)$ & 1.64 & 1.79 \\
& $112.10(4)$ & $159.40(2)$ & 3.25 & 3.34 \\
& $110.84(8)$ & $156.93(4)$ & 3.92 & 4.03 \\
& $116.23(3)$ & $168.18(2)$ & 1.28 & 1.33 \\
& $110.09(3)$ & $154.90(2)$ & 4.51 & 4.63 \\
& $107.71(3)$ & $150.12(2)$ & 6.07 & 6.24 \\
& $105.88(3)$ & $146.40(2)$ & 7.47 & 7.68 \\
& $104.46(4)$ & $143.62(3)$ & 8.64 & 8.90 \\
\hline \hline
\end{tabular}

patterns were recorded on two isotherms, at 300 and $80 \mathrm{~K}$. The patterns were refined using FullProf [16], and a statistical accuracy of typically $4 \times 10^{-4} \AA$ on the lattice parameters of both $\mathrm{NaCl}$ and $\mathrm{Pb}$ was achieved (see Table I). Figure 1 shows typical diffraction patterns at $80 \mathrm{~K}$ and two different pressures,

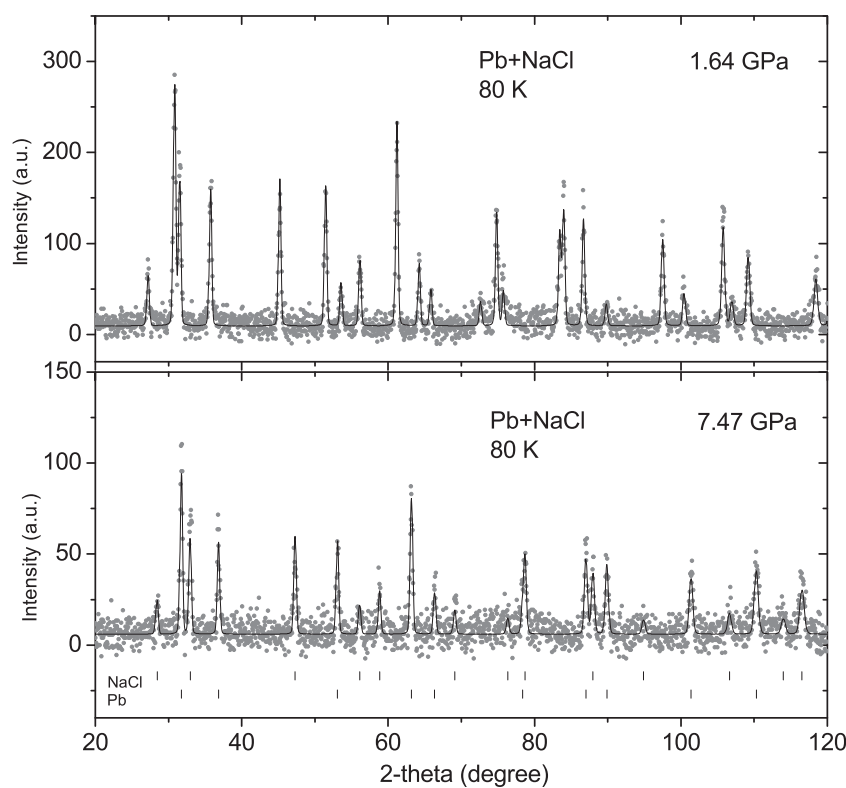

FIG. 1. Neutron-diffraction patterns at $80 \mathrm{~K}$ (as measured, i.e., without background subtraction), at low and high pressure. The lines through the data (dots) are results of Rietveld fits; tick marks below indicate positions of Bragg reflections.

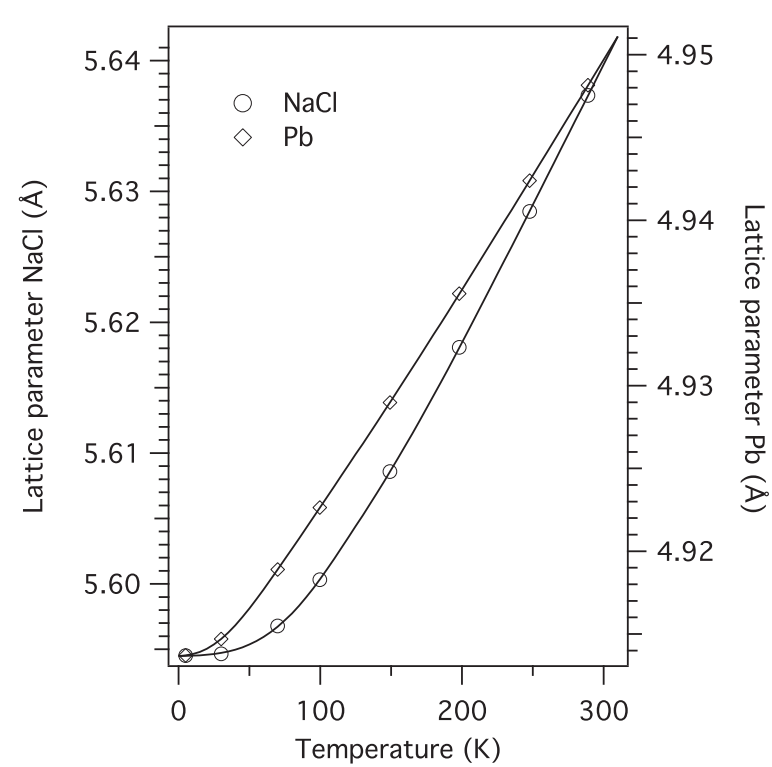

FIG. 2. Measured thermal expansion of $\mathrm{Pb}$ and $\mathrm{NaCl}$ in the 4 $300-\mathrm{K}$ range, at ambient pressure.

along with corresponding fits to the data. Note the absence of any detectable peak broadening.

To obtain accurate ambient pressure lattice parameters as a function of temperature down to $4 \mathrm{~K}$, as well as corresponding thermal expansion coefficients (which are needed for the calculation of the temperature dependence of $B_{0}$ and $B_{0}^{\prime}$ discussed in Sec. IIID), additional diffraction measurements were carried out on a powder mixture of $\mathrm{NaCl}$ and $\mathrm{Pb}$, using the same instrument and a standard ("orange") cryostat with $\mathrm{He}$ as thermal exchange gas. Results are shown in Fig. 2.

\section{ANALYSIS AND DISCUSSION}

\section{A. EOS models}

For each isotherm (300 and $80 \mathrm{~K}$ ) four commonly used EOS forms were considered for fitting the measured $\mathrm{V}(\mathrm{P})$ data. With the definitions

$$
X=\left[V / V_{0}\right]^{1 / 3},
$$

the bulk modulus at ambient pressure

$$
B_{0}=-\left(\frac{\partial P}{\partial \ln V}\right)_{0},
$$

and its pressure derivative at ambient pressure

$$
B_{0}^{\prime}=\left(\frac{\partial B_{0}}{\partial P}\right)_{0},
$$

these are

(1) a simple Murnaghan equation [17],

$$
P(V)=\frac{B_{0}}{B_{0}^{\prime}}\left[X^{-3 B_{0}^{\prime}}-1\right] ;
$$

(2) a third-order Birch equation [18], also called a third-order Birch-Murnaghan EOS,

$$
P(V)=\frac{3}{2} B_{0}\left[X^{-7}-X^{-5}\right]\left[1-\frac{3}{4}\left(4-B_{0}^{\prime}\right)\left(X^{-3}-1\right)\right] ;
$$


(3) a Rydberg-Vinet EOS [19], in the literature mostly called a "Vinet" EOS,

$$
P(V)=\frac{3 B_{0}}{X^{2}}(1-X) \exp \left[\frac{3}{2}\left(B_{0}^{\prime}-1\right)(1-X)\right] ;
$$

and

(4) Holzapfel's AP1 EOS [20], derived from an adapted polynomial expansion, formerly denoted also as H11 [3],

$$
P(V)=\frac{3 B_{0}}{X^{5}}(1-X) \exp \left[\frac{3}{2}\left(B_{0}^{\prime}-3\right)(1-X)\right] .
$$

Although it is not clear which of these forms is the most "universal," there is a general agreement that the Murnaghan EOS [Eq. (3.4)] has major deficiencies and is not able to reproduce reliably compressions typically beyond $5 \%$ in volume $[20,21]$. For this reason, in this paper we will discuss an analysis using only Eqs. (3.5)-(3.7). Since the difference between these three equations becomes significant only at strong compression $(X \rightarrow 0)[20,21]$, it is expected that they will give essentially the same result when applied in the limited stability range of fcc lead.

\section{B. Initial analysis: Skelton's (Decker's) $\mathrm{NaCl}$ scale}

Initial fits of the $V(P)$ data to Eqs. (3.5)-(3.7) were carried out with all three parameters $\left(V_{0}, B_{0}\right.$, and $\left.B_{0}^{\prime}\right)$ adjustable and pressure values obtained from Decker's EOS of $\mathrm{NaCl}$, extended to low temperatures by Skelton et al. [10]. These gave $B_{0} / B_{0}^{\prime}$ values at $298 \mathrm{~K}$ of $40.62(67) \mathrm{GPa} / 5.49$ (24) [Eq. (3.5)], 40.46(66)/5.65(23) [Eq. (3.6)], and 40.53(66)/5.57(23) [Eq. (3.7)], i.e., values which are in good agreement with each other but approximately $3.2 \%$ lower than the value of 41.89 GPa determined by Miller and Schuele from ultrasonic measurements [5]. To judge the insight provided by these results, we can compare them to the $80-\mathrm{K}$ fits, which give $B_{0} / B_{0}^{\prime}$ values of $42.95(1.4) / 6.24(44), 42.83(1.35) / 6.34(39)$, and $42.92(1.34) / 6.26(39)$; i.e., on the surface, our results indicate an increase of $B_{0}^{\prime}$ at low temperatures. This is however physically unrealistic, since while it is well known that $B_{0}^{\prime}$ has a small temperature dependence it is of opposite sign to that of $B_{0}$, i.e., $\partial B_{0}^{\prime} / \partial T>0$. We conclude that the apparent increase of $B_{0}^{\prime}$ upon cooling is an artefact related to the simultaneous increase of $B_{0}$. The strong correlation between $B_{0}$ and $B_{0}^{\prime}$ is a well-known handicap when fitting $V(P)$ data and can easily be demonstrated by imposing $B_{0}^{\prime}$ values between 4.5 and 5.5 and observing the shift in $B_{0}$.

In the case of fcc lead with its relatively small pressure range of stability, diffraction experiments can hardly be expected to further improve the accuracy at which $B_{0}^{\prime}$ may be determined, even if data of better statistics were available, and previous synchrotron data [3] on $\mathrm{Pb}$ demonstrate this very well. The strategy we have chosen instead is to constrain the $B_{0}^{\prime}$ value to the one reported from ultrasonic measurements, a technique which determines $B_{0}^{\prime}$ and $B_{0}$ independently, which gives $B_{0}^{\prime}$ values which we consider the most trustworthy. Using Miller and Schuele's isothermal $B_{0}^{\prime}$ data [5] measured at $298 \mathrm{~K}$ $\left(B_{0}^{\prime}=5.72\right)$ and at $195 \mathrm{~K}\left(B_{0}^{\prime}=5.61\right)$, we extrapolate $B_{0}^{\prime}=5.50$ at $80 \mathrm{~K}$ using a procedure explained further below. We note that these numbers are in good agreement with what is expected from Steinberg's estimates of $B_{0}^{\prime}$ across the different elements, which predict values between 5.5 and 6 (though most of the data compiled in [23] are in fact also derived from ultrasonic data). With these choices of $B_{0}^{\prime}$, we obtain $B_{0}$ values for the three EOS of (in the above order) $B_{0}=40.02(21), 40.25(20)$, and 40.13(21) GPa at $298 \mathrm{~K}$ and $B_{0}=45.30(44), 45.63(48)$, and 45.48(46) GPa at $80 \mathrm{~K}$.

There are two observations. First, at $298 \mathrm{~K}$ all three $B_{0}$ values are on average $4.2 \%$ smaller than the isothermal ultrasonic value, $B_{0}=41.89 \mathrm{GPa}$. Second, the increase of $B_{0}$ between 298 and $80 \mathrm{~K}$ is $13.3 \%$, a result which is independent of the choice of the EOS. Although this increase is almost one order of magnitude larger than what is expected from $\mathrm{x}$-ray-diffraction measurements [8], it is considerably more reasonable on the grounds of semiempirical rules based on Born's theory of lattice dynamics. Fürth [6] pointed out that one would expect

$$
\left(\frac{1}{B_{0}} \frac{\partial B_{0}}{\partial T}\right)=-8.4\left(\frac{1}{V_{0}} \frac{\partial V_{0}}{\partial T}\right)
$$

which is indeed valid to within $\pm 13 \%$ for $\mathrm{Cu}, \mathrm{Pt}, \mathrm{Fe}, \mathrm{Al}$, and $\mathrm{Ag}$. With the data for the thermal expansion of lead (Table I) the expected increase between 300 and $80 \mathrm{~K}$ is $15 \%$, i.e., reasonably close to our measured change.

\section{Final analysis: Brown's $\mathrm{NaCl}$ scale}

The systematically lower value of $B_{0}$ at $298 \mathrm{~K}$ which emerges from both the free fits to the neutron data, as well as fits constrained to the ultrasonic value $B_{0}^{\prime}=5.72$, is for practical purposes (i.e., use as a pressure marker) acceptable, but nevertheless unsatisfying. A possible explanation was hence sought in the choice of the $\mathrm{NaCl}$ pressure scale which was used to determine the pressures. Brown [11] proposed a revised EOS which at $10 \mathrm{GPa}$ deviates by approximately $3 \%$ from the pressure expected from Decker's 1971 standard [9] and hence also Skelton's low- $T$ extension [10] used in our analysis

TABLE II. Normalized volumes $\left(\mathrm{cm}^{3} / \mathrm{g}\right)$ and pressures (GPa) at $T=298$ and $80 \mathrm{~K}$, respectively, using Brown's $\mathrm{NaCl}$ pressure scale extended to low temperatures [22] $\left[v_{\text {norm }}\left(\mathrm{cm}^{3} / \mathrm{g}\right)=V_{\mathrm{Pb}}\left(\AA^{3}\right) \times\right.$ $\left.2.5761 \times 10^{-3}\right]$. Note: the $298-\mathrm{K}$ values deviate slightly from those published in [11], though the calculations are based on the same algorithm and the same input parameters. The reason for this is unknown.

\begin{tabular}{lrrrrr}
\hline \hline$v_{\text {norm }}$ & $T=298 \mathrm{~K}$ & $T=80 \mathrm{~K}$ & $v_{\text {norm }}$ & $T=298 \mathrm{~K}$ & $T=80 \mathrm{~K}$ \\
\hline 0.3618 & 10.80 & 10.35 & & & \\
0.3663 & 9.94 & 9.48 & 0.4161 & 3.28 & 2.76 \\
0.3709 & 9.13 & 8.67 & 0.4206 & 2.86 & 2.34 \\
0.3754 & 8.37 & 7.90 & 0.4251 & 2.47 & 1.95 \\
0.3799 & 7.66 & 7.18 & 0.4297 & 2.11 & 1.57 \\
0.3844 & 6.98 & 6.50 & 0.4342 & 1.76 & 1.22 \\
0.3867 & 6.66 & 6.18 & 0.4364 & 1.59 & 1.05 \\
0.3889 & 6.35 & 5.87 & 0.4387 & 1.42 & 0.88 \\
0.3935 & 5.76 & 5.27 & 0.4432 & 1.11 & 0.57 \\
0.3980 & 5.20 & 4.70 & 0.4478 & 0.82 & 0.27 \\
0.4025 & 4.67 & 4.17 & 0.4523 & 0.54 & -0.01 \\
0.4070 & 4.18 & 3.67 & 0.4568 & 0.28 & \\
0.4116 & 3.71 & 3.20 & 0.4613 & 0.04 & \\
\hline \hline
\end{tabular}


TABLE III. Measured parameters of the equation of state of $\mathrm{Pb}$ at $T=298$ and $80 \mathrm{~K}$ for the three EOS formalisms using Brown's $\mathrm{NaCl}$ pressure scale extended to low temperatures [22] (i.e., Table II, and $B_{0}^{\prime}$ constrained to the ultrasonic values [5], i.e., 5.72 and 5.50, respectively).

\begin{tabular}{lccc}
\hline \hline & & $T=298 \mathrm{~K}$ & $T=80 \mathrm{~K}$ \\
\hline Third-order Birch & $B_{0}$ & $41.01(20)$ & $46.67(50)$ \\
Rydberg-Vinet & $B_{0}$ & $41.25(20)$ & $47.00(52)$ \\
Holzapfel AP1 & $B_{0}$ & $41.13(20)$ & $46.85(51)$ \\
& $B_{0}^{\prime}$ & 5.72 & 5.50 \\
\hline \hline
\end{tabular}

described above. This is typically the difference between the bulk moduli determined by our neutron experiments and other ultrasonic studies, i.e., the choice of the pressure standard is indeed significant.

Brown's $\mathrm{NaCl}$ pressure scale, which so far had only covered temperatures above $300 \mathrm{~K}$, was hence extended to low temperatures using the original algorithm described in [22] (see Table II). The results of fits to the three EOS and the two pressure scales are summarized in Table III, and fits to the measured $V / V_{0}(P)$ data are shown in Fig. 3. It is seen that with this revised $\mathrm{EOS}$ for $\mathrm{NaCl}$ the bulk moduli at $298 \mathrm{~K}$ are 41.01(20), 41.25(20), and 41.13(20) GPa. These values are on average within $\approx 1.8 \%$ of the value $B_{0}=41.89 \mathrm{GPa}$ determined by isothermal ultrasonic measurements, and so the discrepancy

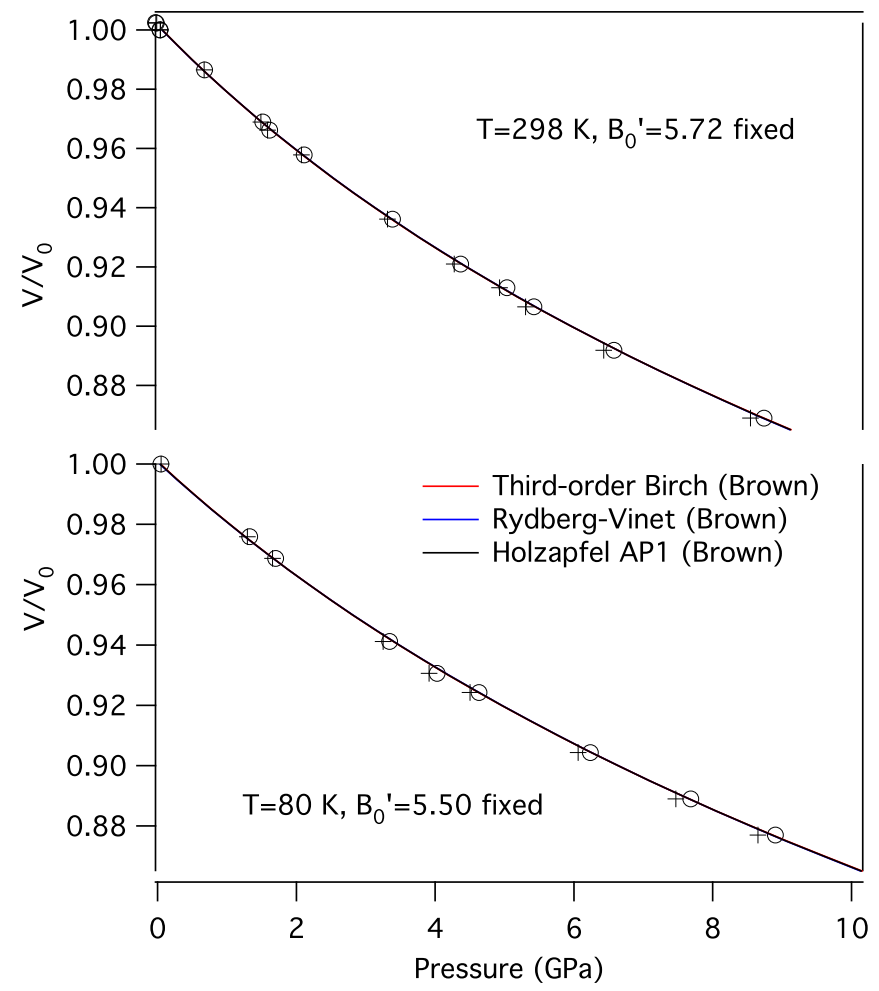

FIG. 3. (Color online) Fits of the experimental data (o) at $T=$ 298 and $80 \mathrm{~K}$ with third-order Birch, Vinet, and Holzapfel AP1 equations of state, respectively (indistinguishable in figure), using Brown's $\mathrm{NaCl}$ pressure scale [11] and its extension to low temperatures [22] (i.e., Table II). Symbols (+) according to Skelton's pressure scale [10]. between the neutron and ultrasound results is reduced by a factor of 2.3 when using the revised $\mathrm{EOS}$ for $\mathrm{NaCl}$. We think that this agreement is not fortuitous but instead a strong indication that Brown's revised $\mathrm{NaCl}$ pressure scale is more accurate, probably even better than $1 \%$ in pressure at $10 \mathrm{GPa}$. This conclusion is supported by combined x-ray-diffraction and ultrasonic measurements of the bulk modulus of $\mathrm{MgO}$, carried out in a multianvil cell [24]. These measurements, which determine $B_{0}$ without the need of a pressure scale, find bulk moduli which are systematically $3-8 \%$ larger compared to measurements where pressures were determined by Decker's $\mathrm{NaCl}$ scale or by ruby fluorescence. Similar to our conclusions, the authors suggest that the source of the difference must be sought in Decker's $\mathrm{NaCl}$ pressure scale.

\section{Full 0-300-K equation of state}

For practical applications, such as using lead as a pressure calibrant, and also for the comparison between our results and first-principles calculations discussed in the next chapter, it is desirable to have bulk moduli over the full $0-300-\mathrm{K}$ temperatures range, and not only at 80 and $298 \mathrm{~K}$. To this end we use a functional temperature dependence for $B_{0}(T)$ and $B_{0}^{\prime}(T)$ as given by the Vinet formalism [19] and scaled it in such a way that $B_{0}^{\prime}$ at 298 and $195 \mathrm{~K}$ reproduce the measured ultrasonic values and $B_{0}$ at 298 and $80 \mathrm{~K}$ reproduce the measured neutron data [25]. The
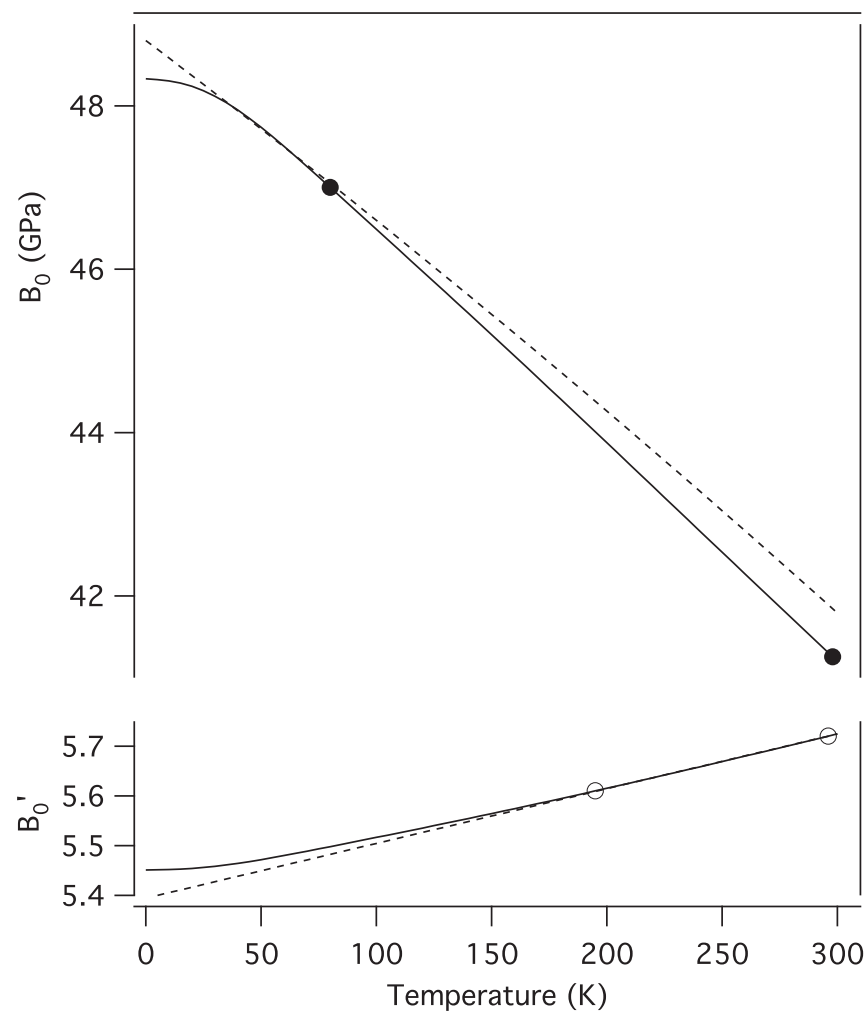

FIG. 4. Temperature dependence of $B_{0}$ and $B_{0}^{\prime}$. The symbols are measured values: $\circ$, ultrasonic data [5]; $\bullet$, our neutron data. The solid lines are interpolations based on a Vinet-type analytical expression [Eqs. (4.5) and (4.6) in [19]] which also includes the measured thermal expansion shown in Fig. 2. Dashed lines refer to temperature dependencies reported by Fortes et al. [26]. 
TABLE IV. Final $B_{0}, B_{0}^{\prime}$ values to be used with the Rydberg-Vinet EOS [Eq. (3.6)] as shown in Fig. 4. See text for details.

\begin{tabular}{lccccc}
\hline \hline$T(\mathrm{~K})$ & $B_{0}(\mathrm{GPa})$ & $B_{0}^{\prime}$ & $T(\mathrm{~K})$ & $B_{0}(\mathrm{GPa})$ & $B_{0}^{\prime}$ \\
\hline 0 & 48.33 & 5.45 & 160 & 44.94 & 5.57 \\
20 & 48.24 & 5.45 & 180 & 44.41 & 5.59 \\
40 & 47.95 & 5.46 & 200 & 43.87 & 5.62 \\
60 & 47.50 & 5.48 & 220 & 43.34 & 5.64 \\
80 & 47.00 & 5.50 & 240 & 42.80 & 5.66 \\
100 & 46.49 & 5.52 & 260 & 42.27 & 5.68 \\
120 & 45.97 & 5.54 & 280 & 41.73 & 5.70 \\
140 & 45.46 & 5.55 & 300 & 41.20 & 5.72 \\
\hline \hline
\end{tabular}

results of this interpolation scheme are shown in Fig. 4, and Table IV gives the corresponding numerical values. It can be used as a "look-up" table for determining pressures from measured lattice constants at various temperatures through corresponding values of $B_{0}(T)$ and $B_{0}^{\prime}(T)$.

\section{E. Potential systematic errors}

The errors cited so far for $B_{0}$ include only statistical errors from the refinements. Potential systematic errors could arise from pressure inhomogeneities, i.e., (a) uniaxial pressure components or (b) the so-called Lamé-effect, i.e., the phenomenon that in a compressed composite powder the individual components can be subjected to slightly different pressures [27]. The former effect is most likely negligible since both $\mathrm{Pb}$ and $\mathrm{NaCl}$ would feel the same uniaxial pressure, i.e., the analysis would not be affected by it. Pressure inhomogeneities from the latter effect can be estimated [27] to cause a pressure difference of a factor of $\left(1+3 \mu_{1} / 4 B_{1}\right) /\left(1+3 \mu_{1} / 4 B_{2}\right)$, where $\mu$ and $B$ are, respectively, the shear strengths and bulk moduli, " 1 " refers to $\mathrm{NaCl}$, and " 2 " refers to $\mathrm{Pb}$. Taking values for $\mu$ from [14] we find a factor of 1.01 ; i.e., the pressure of lead would be at most $1 \%$ higher than we assume from the $\mathrm{NaCl}$ marker. It therefore cannot explain the $4 \%$ difference discussed above, but would in fact bring the corrected bulk modulus even closer to the ultrasonic value. To conclude, the estimated error in $B_{0}$ seems to be $1 \%$ or less.

\section{FIRST-PRINCIPLES RESULTS}

The availability of accurate EOS data at $0 \mathrm{~K}$ allows for a comparison with results obtained from $a b$ initio calculations based on the density-functional theory (DFT) [28]. The computational approach also allows the identification of the relevant mechanisms that contribute to the quantities studied and an insight into their relative importance. A particular question of interest is the role of spin-orbit (SO) coupling. It is known that for heavy atoms this mechanism can influence all quantities which derive from the electronic properties of the system. A few theoretical studies have already addressed the dynamical properties of $\mathrm{Pb}$ [29-33], using different approximations and with various degrees of accuracy. Most often these studies were conducted in the context of phonon dispersions; a few of them also accounted for SO coupling [29,32,33]. In this part of the work we attempt to establish quantitatively the influence of SO coupling in lead on the $P(V)$ EOS and related quantities.

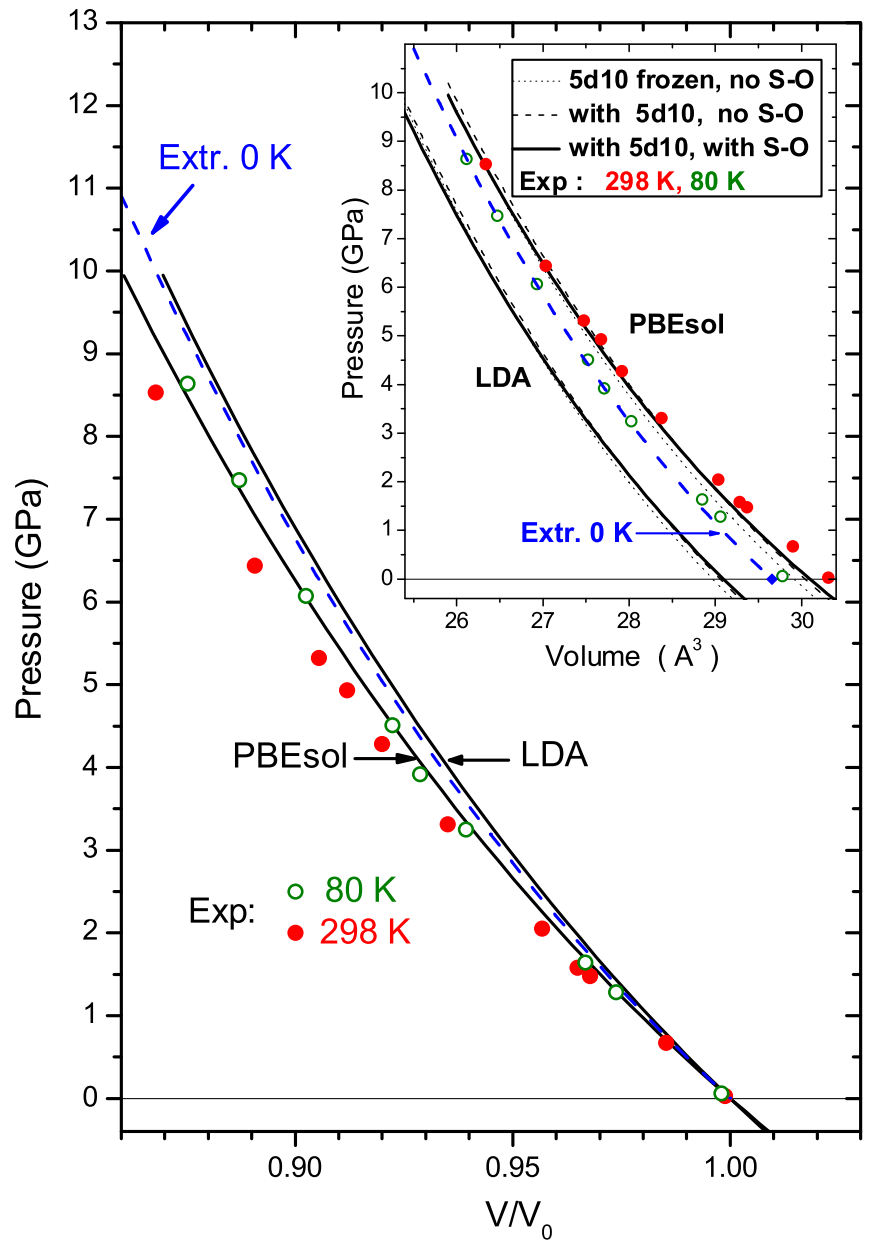

FIG. 5. (Color online) Calculated pressure (solid lines) and experimental data (symbols) vs $V / V_{0}$. The values of $V_{0}$ used are $30.0956 \AA^{3}$ (PBEsol), 29.0945 $\AA^{3}$ (LDA), $30.346 \AA^{3}$ (exp., $298 \mathrm{~K}$ ), $29.839 \AA^{3}$ (exp., $80 \mathrm{~K}$ ), and $29.658 \AA^{3}$ (exp., $0 \mathrm{~K}$ ) and correspond to $1 / 4$ of the cubic $V_{\mathrm{Pb}}$ of Table I. Inset: Pressure calculated using DFT and the LDA- and PBEsol forms for exchange correlation. The dotted, broken, and solid lines demonstrate the effect of relaxation of the semicore $5 d^{10}$ electrons and the contribution of the spin-orbit coupling. The blue dashed lines are the experimental extrapolations to $T=0$.

The calculations were performed in the plane-wave basis using projector augmented waves [34] and VASP codes [35]. We applied the DFT within both the local-density approximation (LDA) [36] and generalized gradient approximation (GGA) [37]; for the latter we chose the form PBEsol [38]. In order to judge the importance of the SO interaction we compare its contribution with the influence of relaxation of the semicore $5 d^{10}$ electrons. The calculated pressures were obtained [39] within the DFT formalism "directly" by using the stress theorem [40]. Unlike in the experiment, we also obtained the $E(V)$ EOS, of which $P(V)$ is the first derivative.

In the inset of Fig. 5 we show the results of the $P(V)$ calculations obtained within the two approximations for exchange-correlation (XC): first, with frozen semicore, i.e., with four valence electrons per $\mathrm{Pb}$, then with the semicore relaxed, with all $5 d^{10}$ electrons included and treated as valence 
TABLE V. EOS parameters for $\mathrm{Pb}$ at $T=0$ derived from the DFT-calculated $P(V)$ data of Fig. 5 and fitted to the Vinet-Rydberg Eq. (3.6). Results are based on 46-50 volumes in the interval (0.871.02) $V_{0}$ and the uncertainties quoted correspond to $90 \%$ confidence range. "EOS spread" is the uncertainty when fits to the other two EOS functions [Eqs. (3.5) and (3.7)] are also considered. Volumes $V_{0}$ refer to $1 / 4$ of the conventional cubic cell. "Earlier calculations" correspond to Murnaghan fits of the calculated $E(V)$ EOS; SR and FR stand for "scalar relativistic" and "full relativistic" treatment, and ZP stands for the zero-point vibrations contribution to the free energy.

\begin{tabular}{|c|c|c|c|}
\hline $\begin{array}{l}\text { Approximations } \\
\text { and the terms included }\end{array}$ & $\begin{array}{c}V_{0} \\
\left(\AA^{3}\right)\end{array}$ & $\begin{array}{c}B_{0} \\
(\mathrm{GPa})\end{array}$ & $B_{0}^{\prime}$ \\
\hline PBEsol, $5 d^{10}$ frozen, no SO & $29.9106(7)$ & $48.38(4)$ & $5.08(1)$ \\
\hline PBEsol, with $5 d^{10}$, no SO & $30.0411(4)$ & $47.95(4)$ & $4.95(1)$ \\
\hline PBEsol, with $5 d^{10}$, with SO & $30.0955(3)$ & $45.65(2)$ & $5.05(1)$ \\
\hline PBEsol, EOS spread & \pm 0.0001 & \pm 0.1 & \pm 0.1 \\
\hline LDA, $5 d^{10}$ frozen, no SO & 28.9703(4) & $53.37(2)$ & $5.07(1)$ \\
\hline LDA, with $5 d^{10}$, no SO & 29.0563(7) & $52.89(4)$ & $4.92(1)$ \\
\hline LDA, with $5 d^{10}$, with SO & 29.0943(9) & $50.53(6)$ & $4.99(2)$ \\
\hline LDA, EOS spread & \pm 0.0002 & \pm 0.1 & \pm 0.1 \\
\hline \multicolumn{4}{|c|}{ Earlier calculations } \\
\hline$[32], \mathrm{LDA}, 5 d^{10}$, no SO & 29.121 & 51.9 & \\
\hline [32], LDA, $5 d^{10}$, with SO & 29.254 & 49.7 & \\
\hline [29], LDA, $5 d^{10}, \mathrm{SR}$ & 29.13 & 52.0 & \\
\hline [29], LDA, $5 d^{10}$, FR & 29.13 & 49.6 & \\
\hline [31], LDA, ZP contribution & $\approx+0.012$ & $\approx-1.0$ & \\
\hline \multicolumn{4}{|c|}{ Experiment, $T=0$} \\
\hline This work: Table IV and text & 29.6578 & 48.33 & 5.45 \\
\hline
\end{tabular}

electrons. The latter calculations are then modified by also accounting for the SO coupling.

Figure 5 (inset) demonstrates the usual under- and overestimation of $V_{0}$ by the LDA and GGA, i.e., respectively, -1.9 and $+1.5 \%$ when compared with the experimental $T=0$ value for $V_{0}$. The figure allows us both to judge the effect of relaxation of the $5 d^{10}$ semicore, and to account for the SO coupling: the difference between the dotted, broken, and solid lines is small, which suggests a weak influence of the above two factors, both on the LDA and GGA results.

In order to judge these contributions quantitatively we fitted the calculated $P(V)$ data to the three EOS models given by Birch [18], Vinet et al. [19], and Holzapfel [20], discussed in Sec. III A, and expressed the $P(V)$ variation in terms of $V_{0}, B_{0}, B_{0}^{\prime}$ (Table V). The results based on the $P(V)$-EOS are coherent with the fits of $E(V)$ (not shown) performed in parallel and are in line with previous calculations [29,32]. Perusal of Table $\mathrm{V}$ shows that the relaxation of the semicore leads to a slight increase of $V_{0}(0.3-0.4 \%)$. On the other hand, the bulk modulus of $B_{0}$ is modified more significantly by the inclusion of SO coupling $(\approx-5 \%)$ and just $\approx-0.9 \%$ when accounting for the semicore. Semicore relaxation also reduces the value of $B_{0}^{\prime}(-3 \%$, in both LDA and PBEsol), while the influence of SO coupling on $B_{0}^{\prime}$ could not be established unambiguously. In terms of absolute values, most of these modifications are small, which only stresses the importance of a consistent treatment of the entries in Table $\mathrm{V}$.

The values of $V_{0}, B_{0}, B_{0}^{\prime}$ given in Table $\mathrm{V}$ correspond to adjusting the calculated $P(V)$ data to the Vinet EOS, and the "EOS spread" entry indicates variations when also the Birch and Holzapfel forms are considered. The uncertainty arising from the choice of a particular model EOS is hence not negligible but, for $V_{0}$ and $B_{0}$, it is smaller than the effect of any of the two "physical" mechanisms discussed above.

None of our DFT calculations take into account the zeropoint vibrations term in free energy, but estimates for the associated changes in $V_{0}, B_{0}$ are given in [31], which we include in Table V. Not surprisingly, the effect on $V_{0}$ is negligible for a heavy atom such as $\mathrm{Pb}$, but the calculated $B_{0}$ decreases by $\approx 2 \%$.

In order to facilitate the comparison between calculations and experiments in Fig. 5 we plot the same data against the relative volume $V / V_{0}$. The agreement between the $80-\mathrm{K}$ data with the $(T=0)$ calculations within the PBEsol framework is remarkably good at small and moderate compressions $\left(V / V_{0}=0.9-1.0\right)$ where the slopes are still controlled mainly by $B_{0}$. The small differences at $V / V_{0} \leqslant 0.9$ reflect the minor disagreement between the calculated and experimental $B(V)$ - a consequence of the lower $B_{0}^{\prime}$ predicted by the DFT. Nevertheless the $T=0$ data constructed from the $B_{0}(T \rightarrow 0)$ and $B_{0}^{\prime}(T \rightarrow 0)$ values shown in Table IV are in better agreement with the LDA calculations. We observe that the $\approx 5 \%$ too high LDA prediction for the initial slope is partly compensated, at higher pressures, by the $\approx 8 \%$ too low LDA result for $B_{0}^{\prime}$.

Obviously it is the choice of the approximation for $\mathrm{XC}$ that plays a crucial role in describing the EOS and other physical properties (see, e.g., [41] for an attempt to assess the performance of five types of XC in 24 solid metals and nonmetals). The results shown here seem to suggest that for the prediction of the EOS in $\mathrm{Pb}$ the bracketing provided by the PBEsol and LDA approaches provides the closest boundaries to the experiment, thus setting the uncertainty of the DFT predictions for $P\left(V / V_{0}\right)$ at about $\pm 5 \%$.

\section{SUMMARY}

We have reported a detailed investigation of the lowtemperature EOS of $\mathrm{Pb}$ in the 0-10-GPa range. The data have various implications:

(1) We believe that they represent the hitherto most accurate dataset for metrological purposes, i.e., for using $\mathrm{Pb}$ as a pressure manometer. For practical purposes we recommend any of the three equation-of-state forms given by Eqs. (3.5)(3.7) with the temperature dependence of the bulk modulus $B_{0}$ and its zero-pressure derivative $B_{0}^{\prime}$ given in Table IV. Our EOS gives pressure values which coincide at $300 \mathrm{~K}$ and $10 \mathrm{GPa}$ within $0.6 \%$ of the most recent $\mathrm{x}$-ray results [8], where pressure was also determined by a $\mathrm{NaCl}$ marker, and at $195 \mathrm{~K}$ to within $1 \%$ of the ultrasonic data provided by Miller and Schuele [5] (assuming these can be extrapolated from 0.13 to $10 \mathrm{GPa}$ ). As for the EOS proposed by Fortes et al. [26] which was constructed using the same ultrasonic and x-ray data, it overestimates pressures at $300 \mathrm{~K}$ and $10 \mathrm{GPa}$ by $2 \%$. This deviation reduces at low temperatures and reverses below $50 \mathrm{~K}$ to give an underestimation by $0.8 \%$ at $0 \mathrm{~K}$.

(2) The pressures used in this work are based on Brown's $1999 \mathrm{NaCl}$ scale [11]. The rather good agreement between our neutron data and the above-cited ultrasonic and x-ray work 
degrades significantly if we use Decker's 1977 scale in our analysis. We believe that this provides significant experimental evidence that Brown's $\mathrm{NaCl}$ scale is more accurate and supports similar conclusions derived from measurements on $\mathrm{MgO}$ [24]. It would be useful to carry out similar studies on other materials.

(3) The present results made it possible to confront lowtemperature experimental data close to $T=0$ with calculations and thereby allow conclusions on the accuracy of some approximations used therein. While it is impossible to make general recommendations, it appears from this work that, among the plethora of approximations, PBEsol and LDA are the most appropriate for the predictions of bulk moduli for $\mathrm{Pb}$ and maybe even other heavy elements. Depending on technical ingredients (Table $\mathrm{V}$ ), its accuracy for $0-\mathrm{K} B_{0}$ is better than $3 \%$, but it systematically underestimates $B_{0}^{\prime}$ by $\approx 8 \%$. As for the $P(V)$ relation, a conservative estimate for the accuracy of the DFT predictions is the width of the LDA-GGA bracketing, which, in the present case, amounts to $\pm 5 \%$ of $P$ at any given $V / V_{0}$ (see Fig. 5). From a general point of view, our data demonstrate that EOS parameters from experiment and theory need to be determined at similar temperatures if a meaningful comparison is sought.

\section{ACKNOWLEDGMENTS}

This work is based on experiments performed at the Swiss spallation neutron source SINQ, Paul Scherrer Institut, Villigen, Switzerland, and has been supported by the European Commission under the Seventh Framework Programme through the "Research Infrastructures" action of the "Capacities" Programme, NMI3-II Grant No. 283883. We are very grateful to Prof. J. M. Brown for providing the low-temperature values for his $\mathrm{NaCl}$ pressure scale. We are very grateful to Prof. J. M. Brown for providing the low temperature values for his $\mathrm{NaCl}$ pressure scale and Karl Syassen for making available Datlab with its algorithms for fitting EOS-models. K.K. benefited from access to the High Performance Computing resources of IDRIS (Orsay, France) under the allocation CP09-0208 made by GENCI. J.W. gratefully acknowledges financial support from the Swiss NCCR program MaNEP.
[1] H. K. Mao, Y. Wu, J. F. Shu, J. Z. Hu, R. J. Hemley, and D. E. Cox, Solid State Commun. 74, 1027 (1990).

[2] Y. K. Vohra and A. L. Ruoff, Phys. Rev. B 42, 8651 (1990).

[3] O. Schulte and W. B. Holzapfel, Phys. Rev. B 52, 12636 (1995).

[4] D. L. Waldorf and G. A. Alers, J. Appl. Phys. 33, 3266 (1962).

[5] R. A. Miller and D. E. Schuele, J. Phys. Chem. Solids 30, 589 (1969).

[6] R. Fürth, Proc. Cambr. Phil. Soc. 37, 34 (1941).

[7] H. Ledbetter, Phys. Status Solidi B 181, 81 (1994).

[8] A. Kuznetsov, V. Dimitriev, L. Dubrovinsky, V. Prakapenka, and H.-P. Weber, Solid State Commun. 122, 125 (2002).

[9] D. L. Decker, J. Appl. Phys. 42, 3239 (1971).

[10] E. F. Skelton, A. W. Webb, S. B. Qadri, S. A. Wolf, R. C. Lacoe, J. L. Feldman, W. T. Elam, E. R. Elam, E. R. Carpenter, Jr., and C. Y. Huang, Rev. Sci. Instrum. 55, 849 (1984).

[11] J. M. Brown, J. Appl. Phys. 86, 5801 (1999).

[12] K. Syassen, High Press. Res. 28, 75 (2008).

[13] S. Klotz, Th. Strässle, G. Rousse, G. Hamel, and V. Pomjakushin, Appl. Phys. Lett. 86, 031917 (2005).

[14] W. F. Sherman and A. A. Stadtmuller, Experimental Techniques in High-Pressure Research (Wiley, New York, 1987).

[15] S. Klotz, J. M. Besson, and G. Hamel, High Press. Res. 26, 277 (2006).

[16] J. Rodríguez-Carvajal, Physica B 192, 55 (1993); http://wwwllb.cea.fr/fullweb/powder.htm.

[17] F. D. Murnaghan, Am. J. Math. 59, 235 (1937).

[18] F. Birch, J. Appl. Phys. 9, 279 (1938).

[19] P. Vinet, J. R. Smith, J. Ferrante, and J. H. Rose, Phys. Rev. B 35, 1945 (1987).

[20] W. B. Holzapfel, High Press. Res. 16, 81 (1998).

[21] K. Syassen, Isothermal EOS Functions for Solids, Max-PlanckInstitut, Stuttgart, (2012) (unpublished).
[22] M. Brown (private communication).

[23] D. J. Steinberg, J. Phys. Chem. Solids 43, 1173 (1982).

[24] B. Li, K. Woody, and J. Kung, J. Geophys. Res. Sol. Earth 111, B11206 (2006).

[25] See Eqs. (4.5) and (4.6) of [19]. Without scale factors of 1.26 and 1.32 the Vinet formalism would underestimate the $T$ dependencies of both $B_{0}^{\prime}$ and $B_{0}$, respectively.

[26] A. D. Fortes et al., High Press. Res. 27, 201 (2007); 32, 337 (2012).

[27] Y. Wang, D. J. Weidner, and Y. Meng, in Properties of the Earth and Planetary Materials at High Pressure and Temperature, Geophysical Monograph Vol. 101, edited by M. H. Manghnani and T. Yagi (American Geophysical Union, Washington D.C., 1998), p. 365.

[28] P. Hohenberg and W. Kohn, Phys. Rev. 136, B864 (1964).

[29] See, e.g., A. Dal Corso, J. Phys. Condens. Matter 20, 445202 (2008) and references therein.

[30] S. de Gironcoli, Phys. Rev. B 51, 6773 (1995).

[31] B. Grabowski, T. Hickel, and J. Neugebauer, Phys. Rev. B 76, 024309 (2007).

[32] R. Heid, K.-P. Bohnen, I. Yu. Sklyadneva, and E. V. Chulkov, Phys. Rev. B 81, 174527 (2010).

[33] A. Dal Corso, Phys. Rev. B 86, 085135 (2012).

[34] P. E. Blöchl, Phys. Rev. B 50, 17953 (1994); G. Kresse and D. Joubert, ibid. 59, 1758 (1999).

[35] G. Kresse and J. Hafner, Phys. Rev. B 47, R558 (1993); G. Kresse, Ph.D. thesis, Technische Universität Wien, 1993; G. Kresse and J. Furthmüller, Comput. Mater. Sci. 6, 15 (1996); Phys. Rev. B 54, 11169 (1996).

[36] W. Kohn and L. J. Sham, Phys. Rev. 140, A1133 (1965).

[37] D. C. Langreth and M. J. Mehl, Phys. Rev. B 28, 1809 (1983); 29, 2310(E) (1984); A. D. Becke, Phys. Rev. A 38, 3098 (1988); J. P. Perdew, J. A. Chevary, S. H. Vosko, K. A. Jackson, M. R. Pederson, D. J. Singh, and C. Fiolhais, Phys. Rev. B 46, 6671 (1992); 48, 4978(E) (1993). 
[38] J. P. Perdew, A. Ruzsinszky, G. I. Csonka, O. A. Vydrov, G. E. Scuseria, L. A. Constantin, X. Zhou, and K. Burke, Phys. Rev. Lett. 100, 136406 (2008); 102, 039902(E) (2009).

[39] The plane-wave cutoff used is $E_{\mathrm{pw}}=400 \mathrm{eV}$, and the k-point sampling was performed on the $24 \times 24 \times 24$ Monkhorst-Pack [42] meshes (1300 irreducible $\mathbf{k}$ points). In order to account for the metallic nature of lead we used the Gaussian smearing technique [43] with $\sigma=0.2 \mathrm{eV}$.
[40] O. H. Nielsen and R. M. Martin, Phys. Rev. Lett. 50, 697 (1983); Phys. Rev. B 32, 3780 (1985).

[41] G. I. Csonka, J. P. Perdew, A. Ruzsinszky, P. H. T. Philipsen, S. Lebègue, J. Paier, O. A. Vydrov, and J. G. Angyán, Phys. Rev. B 79, 155107 (2009).

[42] H. J. Monkhorst and J. D. Pack, Phys. Rev. B 13, 5188 (1976).

[43] M. Methfessel and A. T. Paxton, Phys. Rev. B 40, 3616 (1989). 